\title{
A Continuous Review Inventory System with Lost Sales and Emergency Orders
}

\author{
Michael Haughton, K. P. Sapna Isotupa* \\ School of Business \& Economics Wilfrid Laurier University Waterloo, Ontario, Canada \\ Email: mhaughton@wlu.ca, *sisotupa@wlu.ca
}

How to cite this paper: Haughton, M. and Isotupa, K.P.S. (2018) A Continuous Review Inventory System with Lost Sales and Emergency Orders. American Journal of Operations Research, 8, 343-359.

https://doi.org/10.4236/ajor.2018.85020

Received: May 29, 2018

Accepted: September 8, 2018

Published: September 11, 2018

Copyright $\odot 2018$ by authors and Scientific Research Publishing Inc. This work is licensed under the Creative Commons Attribution International License (CC BY 4.0).

http://creativecommons.org/licenses/by/4.0/

\begin{abstract}
We analyze a continuous review lost sales inventory system with two types of orders-regular and emergency. The regular order has a stochastic lead time and is placed with the cheapest acceptable supplier. The emergency order has a deterministic lead time is placed with a local supplier who has a higher price. The emergency order is not always filled since the supplier may not have the ability to provide the order on an emergency basis at all times. This emergency order has a higher cost per item and has a known probability of being filled. The total costs for this system are compared to a system without emergency placement of orders. This paper provides managers with a tool to assess when dual sourcing is cost optimal by comparing the single sourcing and dual sourcing models.
\end{abstract}

\section{Keywords}

Continuous Review, Inventory, Lost Sales, Emergency Order

\section{Introduction}

In this paper we analyze an inventory system with two types of orders, a regular order and an emergency order under the lost sales framework. Reducing stock out risk by splitting replenishment requirements among multiple suppliers is a sourcing policy that has attracted the attention of academic researchers for more than 20 years. The policy is theoretically appealing for several reasons. First, pooling lead-time uncertainty among several suppliers is a way to reduce the safety stock needed to meet service targets or alternatively, the expected number of backorders for a prescribed level of safety stock. Second, successive deliveries of smaller orders will reduce cycle stock. Third, the incremental ordering cost of the second as subsequent orders may be relatively small in a variety of settings. 
Since the tragic events of September 11, 2001, several security initiatives have been implemented at US international border checkpoints. These aim to minimize the risk of trans-border flows of merchandise being conduits for harm to national security. For many companies delivering products by trucks from Canada to the US, these measures have increased both the mean and variance of border crossing times. Consequently, some US companies (for example the automotive manufacturing industry) that traditionally obtained their raw material from Canada have switched to using just American suppliers or using both a Canadian and an American supplier. In the latter case, companies split their requirements between the less expensive and less lead-time reliable Canadian supplier and the more expensive but more lead-time reliable American supplier. This has boosted interest in research on inventory systems with multiple suppliers and provides the motivation for our research.

In this paper we will compare two inventory policies. The policy we will focus most of our attention on is a $(\mathrm{Q}, \mathrm{R})$ inventory policy with two suppliers-a regular supplier and an emergency supplier. The emergency supplier is used only when the inventory level is dangerously low and a stock out risk is imminent. We consider the case of a manufacturer facing demand that is predictable and occurring at equally spaced time intervals. The lead time for the regular order is probabilistic and highly variable with a high variance due to the unpredictability in border crossing times. When inventory falls to dangerously low levels, an emergency order is placed with a local supplier or with the competition. The order is filled with a certain probability. If the order is filled, it will be filled in a certain fixed amount of time which is deterministic. There is a fixed cost of placing the order which is incurred whether the order is filled or not. The variable cost of the emergency order, which is proportional to the order size, is only incurred if the order is filled. There are various reasons why the emergency order may not be filled. If the emergency order is placed with a local supplier with whom the company does not have a large amount of business, this company may reserve their stock for higher priority customers and choose not to fill the order of this manufacturer. If the order is placed with the competition, then for strategic business reasons, they may choose not to fill that particular order. We will compare the total long run cost rate of this policy to the traditional $(\mathrm{Q}, \mathrm{R})$ inventory policy with predictable demand and lost sales. With the help of numerical examples we provide some situations where dual sourcing with emergency order placements is cost effective when compared to a single sourcing $(\mathrm{Q}$, $\mathrm{R})$ model. There are two main contributions of this paper-1) Determine the long run expected total cost of a $(\mathrm{Q}, \mathrm{R})$ system with emergency orders and lost sales, 2) Provide a method by which to compare a lost sales $(\mathrm{Q}, \mathrm{R})$ system to a lost sales $(\mathrm{Q}, \mathrm{R})$ system with emergency orders and illustrate with numerical examples when the system with emergency orders is cost effective.

Rapid advancements in computer and information technology along with the crucial role of responsiveness as a winning supply chain strategy have boosted interest in continuous review inventory policies. However, the bulk of the in- 
ventory models dealing with multiple suppliers are for the periodic review case. The focus on periodic review is mainly because of the mathematical complexity in dealing with continuous review systems. In this paper we will deal with a continuous review $(\mathrm{Q}, \mathrm{R})$ inventory system with lost sales and two suppliers. By identifying and establishing the equivalence of this model to another mathematical model which is more tractable, we will obtain analytic expressions for the total cost of running the system. We will then compare the two policies numerically in a variety of scenarios and identify situations where a policy with two suppliers performs better than a system with a single supplier.

The paper has 6 sections. The second section consists of literature review of inventory systems for multiple suppliers. Section 3 is the crux of the paper where the mathematical model and detailed analysis are presented. Section 3 is also central in demonstrating how we address what has been heretofore seen as a mathematical difficulty of continuous review $(\mathrm{Q}, \mathrm{R})$ systems. The steady state inventory level distribution is also derived in this section. In Section 4, the expression for the long-run expected cost rate is developed. In Section 5 some numerical examples are presented. Section 6 consists of conclusions and future research.

\section{Literature Survey}

The literature on inventory systems with multiple suppliers can be broadly separated into two classes-one, where replenishment orders are split simultaneously among many suppliers and two, where orders are placed at different times with different suppliers. In this paper we focus on the second case and hence only provide a literature survey of papers of this kind. For information on models of the first type, useful sources are Thomas and Tyworth [1] and the review paper by Minner [2] on multiple supplier inventory models in both the periodic and continuous review cases.

Past related research includes the early papers of Barankin [3] and Neuts [4], which studied periodic review inventory systems with regular and emergency replenishments, where the regular order lead-time is one period and the emergency replenishment is instantaneous. The more recent studies on emergency ordering have all been in the periodic review realm. The latest study of a periodic review system with emergency orders was by Johansen [5]. They study an inventory system with compound Poisson demands and backorders using Markov decision processes. They do not have any analytical comparisons of cost, but based on numerical examples they show that a combination of normal orders and emergency orders yield lower system costs than having no emergency orders.

However, since all these papers focus on periodic review models while we address the continuous review case, we exclude the periodic review models from further consideration in our literature survey. Our literature survey focuses only on continuous review inventory systems with both regular and emergency orders. Our literature survey does not include the literature on inventory systems 
where suppliers are either available or unavailable but no emergency ordering is done during the unavailable period of the suppliers nor does it include the case of transhipment of inventory between two locations. Further we do not present a literature review of two echelon inventory models or simulation studies of inventory models.

Dohi, Kaio and Osaki, S. [6] and Giri and Dohi [7] study continuous review inventory systems where after a fixed amount of time, $t_{o}$, if stock is depleted, an emergency order is placed which arrives after a lead time L1 and if stock is not depleted, a regular order is placed which arrives after a lead time L2. The first paper derives the necessary and sufficient conditions for $t_{o}$ to exist which minimizes the long-run average cost. The second paper they derive the optimal ordering time that minimizes average cost for a fixed ordering quantity model. Bradley [8] analyzed a production-inventory model in which in-house production and a sub-contractor are the inventory replenishment alternatives. Using Brownian motion approximations, the author sought to determine the optimal policy parameters. Allon and Van Miegham [9] studied a continuous review inventory model with dual sourcing. They considered the problem of splitting orders between a responsive and expensive supplier versus a slow but inexpensive supplier. Despite some similarities between their work and ours, there are several important differences. For example, they deal with the case of backorders while we deal with the case of lost sales. Also, in their paper the two orders are placed with the two suppliers simultaneously while in our paper, the order with the expensive supplier is only placed if the stock levels fall dangerously low.

Allen and D'Esopo [10] were the first to consider a continuous review inventory system with emergency orders. They analyzed the standard $(\mathrm{Q}, \mathrm{R})$ inventory model with an additional parameter called the expediting level. Moinzadeh and Schmidt [11] considered the $(S-1, S)$ inventory system with emergency orders. Song and Zipkin [12] extend this model to include the case of multiple suppliers and develop performance evaluation tools for a variety of policies under which the supply system becomes a network of queues. Johansen and Thorstenson [13] adopted the standard $(\mathrm{Q}, \mathrm{R})$ policy for regular orders and an $(\mathrm{s}, \mathrm{S})$ type policy for emergency orders, where $s$ and $S$ depend ingeneral on the time remaining until the receipt of a regular order. They deal with the case of complete backordering and use simulation to obtain the optimal values of the reorder levels and order quantities.

Moinzadeh and Nahmias [14] proposes a very general model with the $(\mathrm{Q}, \mathrm{R})$ system with back orders, two reorder points and two reorder quantities and proposes a heuristic control policy for the case where lead times are deterministic. Their paper assumes that the lead times for both orders are deterministic and that the demand can follow a Poisson or normal distribution. Mohebbi and Posner [15] analyze the model by Moinzadeh and Nahmias [14] under the assumption of compound Poisson demand and non-identical exponentially distributed lead times using the level crossing approach and develop the total cost function. Duran, Gutierrez and Zequeira [16] analyze a system similar to the one 
by Moinzadeh and Nahmias [14]. They analyze a continuous review $(\mathrm{Q}, \mathrm{R})$ system with backorders where the lead time has a fixed component $T$. If the inventory level lies below a threshold level when $\mathrm{T}$ units of time has elapsed since order placement, the order is expedited and arrives after a short but deterministic time and if the inventory level is not below the threshold level, there is a longer deterministic period of time before the order arrives. They present an algorithm to determine the policy parameters that minimize the total cost. The paper most closely related to our paper is the one by Axsater [17] which deals with a (Q, R) inventory system with Poisson demands and emergency ordering. There are four important differences between Axsater [17] and our model. Our paper is motivated by the fact the lead time for the order placed with the cheaper (regular) supplier is highly variable, which necessitates the use of an alternate supplier. Hence in our case only the emergency order has a deterministic lead time and the regular order has a stochastic lead time whereas both orders have deterministic lead time in Axsater [17]. Further in our paper, which has applications to the auto industry, the demand pattern is deterministic since the production lines at most auto companies run on a continuous basis while the demand pattern in Axsater [17] is Poisson. Our paper deals with the situation where the availability of the emergency order is probabilistic whereas in Axsater [17], the assumption is that the emergency order will always get filled. The last difference is that we consider the lost sales case while Axsater [17] considers the backlogging case.

\section{Problem Description and Analysis}

We consider a continuous review $(Q, R)$ inventory system for a US based manufacturer where the demand for the item is predictable and is one unit every $T$ periods; i.e., mean demand per period $=1 \div T$. Note that because at each demand epoch, there is a demand for just one item, the $(Q, R)$ policy is equivalent to the $(s, Q)$ policy and from here on, we will refer to the model as an $(s, Q)$ system. The maximum inventory level is $Q+s$ units. When the inventory level drops to the reorder point, $s$, a regular order of size $Q(Q>s)$ is placed with a Canadian supplier. The assumption that $Q>s$ ensures that there is at most one outstanding regular order at any given time. Because of the highly unpredictable border crossing times, the lead time for the regular order is assumed to be exponentially distributed with rate $\mu$. The system we consider is a lost sales system. Hence if the inventory level drops to zero and there is a demand for the item by a customer, the customer is sent away with his demand unsatisfied. The business is investigating the possibility of procuring stock on an emergency basis from a local supplier or from the competition if the reorder doesn't arrive when the stock level drops to $n$.

The time taken to procure items on an emergency basis from the local supplier or the competition is deterministic and takes $n T$ units of time where $n$ is less than the reorder level, $s$, for obvious reasons. Hence when the stock level drops to $n$, an emergency order of size $s$ is placed with the local supplier or competition. This order has a probability $p$ of being fulfilled. Note that an emergency 
order is placed only at the instant when the stock level drops to $n$ from $n+1$ and not at times when the inventory level is $n$. If the order is filled, it is for the entire $s$ units. The local supplier may guarantee that the stock will be available $100 \mathrm{p} \%$ of the time. In the case of the competition, obviously there will be no guarantees and $100 \mathrm{p} \%$ is the estimated percentage of time that the manufacturer can get the stock from the competition.

The system described above is equivalent to a lost sales $(s, Q)$ inventory system where demands occur once every $T$ units with the following additional conditions. The reorder policy with the Canadian supplier is the same as in the model described in the previous paragraph. The emergency order described in that model is equivalent to an emergency order placed when the inventory level drops to zero which gets replenished with probability $p$. If the order is filled, it is filled instantaneously. In this paper we will model this system as a system with instantaneous replenishment of the emergency order and follow through with the analysis.

In order to determine the long-run expected cost rate, we need to determine the steady state inventory level distribution $P(j)$, where $P(j)$ denotes the steady state probability that inventory level is $j$. In the analysis that follows, $\pi(j)$ denotes the stationary distribution of the embedded Markov chain.

Theorem 1: The steady state inventory level distribution $P(j)$ is given by

$$
\begin{gathered}
P(0)=\frac{\left(\mathrm{e}^{\mu T}-1\right) \pi(0)}{\mu T} \\
P(j)=\frac{\left(\mathrm{e}^{\mu T}-1\right)^{2}}{\mu T(1-p)} \mathrm{e}^{(j-1) \mu T} \pi(0) ; 1 \leq j \leq s \\
P(j)=\frac{\left(\mathrm{e}^{\mu T}-1\right)}{(1-p)} \mathrm{e}^{s \mu T} \pi(0)-\frac{p\left(\mathrm{e}^{\mu T}-1\right)}{(1-p)} \pi(0) ; s+1 \leq j \leq Q-1 \\
P(Q)=\frac{\left(\mathrm{e}^{\mu T}-1\right)}{(1-p)} \mathrm{e}^{s \mu T} \pi(0)-\frac{p\left(\mathrm{e}^{\mu T}-1\right)}{(1-p)} \pi(0)+\pi(0)-\frac{\left(\mathrm{e}^{\mu T}-1\right) \pi(0)}{\mu T} \\
P(j)=\frac{\left(\mathrm{e}^{\mu T}-1\right)}{(1-p)} \mathrm{e}^{s \mu T} \pi(0)-\frac{p\left(\mathrm{e}^{\mu T}-1\right)^{2}}{\mu T(1-p)} \mathrm{e}^{(j-Q-1) \mu T} \pi(0) ; Q+1 \leq j \leq Q+s
\end{gathered}
$$

where

$$
\pi(0)=\frac{1}{1+\frac{Q\left(\mathrm{e}^{\mu T}-1\right)}{1-p} \mathrm{e}^{s \mu T}+\frac{(Q-s) p\left(\mathrm{e}^{\mu T}-1\right)}{1-p}}
$$

Proof: Let $I(t)$ denote the inventory level at time $t$. From our assumptions it is clear that the inventory level process $\{I(t) ; t \geq 0\}$ with state space $E=\{0,1,2, \ldots$, $Q+S\}$ is a semi-regenerative process with the regeneration points being the demand epochs. Let $\left\{\tau_{0}, \tau_{1}, \tau_{2}, \cdots\right\}=\{0, T, 2 T, \cdots\}$ be the successive epochs at which demands occur. If $I_{n}=I\left(\tau_{n}^{-}\right)$, then $(I, \tau)=\left\{I_{n}, \tau_{n} ; n \in N^{0}\right\}$ is a Markov 
renewal process with embedded Markov chain $\left\{I_{n} ; n \in N^{0}\right\}$. Let us define the inventory level distribution as follows:

$$
P(i, j, t)=\operatorname{Pr}\left[I(t)=j \mid I_{0}=i\right]
$$

Then from Markov renewal theory (refer Cinlar (1975)), $P(i, j, t)$ satisfies the following Markov renewal equation:

$$
P(i, j, t)=K(i, j, t)+\sum_{l=0}^{s} \int_{0}^{t} \theta(i, l, u) P(l, j, t-u) \mathrm{d} u
$$

where $\theta(i, j, t)$ is the derivative of the semi-Markov kernel of the Markov renewal process $(I, \tau)$ and is given by

$$
\theta(i, j, t)=\lim _{\Delta \rightarrow 0} P\left[I_{1}=j, t \leq \tau_{1} \leq t+\Delta \mid I_{0}=i\right] / \Delta
$$

and

$$
K(i, j, t)=P\left[I(t)=j, \tau_{1}>t \mid I_{0}=i\right]
$$

The various operating characteristics that are necessary to obtain the long-run expected cost rate of the inventory system can be obtained in terms of the steady state inventory level distribution if it exists.

From Markov renewal theory the steady state distribution of the inventory level exists as the state space is finite and the embedded Markov chain is irreducible. Let $P(j)$ be the steady state inventory level distribution. Then from Cinlar [18], we have

$$
P(j)=\frac{\sum_{i=0}^{s} \pi(i) K^{*}(i, j, 0)}{\sum_{i=0}^{s} \pi(i) m(i)}
$$

where $K^{*}(i, j, 0)$ is the Laplace transform of $K(i, j, t)$ evaluated at zero and $m(i)$ is the mean sojourn time in state $i$. The stationary distribution of the embedded Markov chain is given by $\pi(i)$ and obtained by solving the equations

$$
\pi(j)=\sum_{i} \pi(i) \int_{0}^{\infty} \theta(i, j, t) \mathrm{d} t
$$

and the normalizing condition $\sum_{j} \pi(j)=1$.

Since time between two consecutive (unit) demands is deterministic and equal to $T$,

$$
\sum_{i=0}^{s} \pi(i) m(i)=T
$$

In order to determine the steady state inventory level distribution, we first need to determine $\int_{0}^{\infty} \theta(i, j, t) \mathrm{d} t$ and $\int_{0}^{\infty} K(i, j, t) \mathrm{d} t$.

To determine $\theta(i, j, t)$, we note that the transition points are either demand or replenishment epochs. For example let the inventory level just before a demand occurs be 0 and hence after the demand occurs and before the next demand, the inventory level will either remain at 0 if no replenishment occurs with probability $\mathrm{e}^{-\mu t}$ and the inventory level will reach $Q$ if a replenishment occurs 
with probability $1-\mathrm{e}^{-\mu t}$. In our case, the time between demand epochs is deterministic. Hence $\theta(i, j, t)$ is non-zero only when $t=T$. Denote by $p(i, j)$, $\int_{0}^{\infty} \theta(i, j, t) \mathrm{d} t$. Then $p(i, j)$ are the one step transition probabilities of the Markov chain embedded in the Markov renewal process $(I, \tau)$ and are given by the function $\theta(i, j, T)$.

$$
p(i, j)= \begin{cases}\mathrm{e}^{-\mu T} & i=j=0 ; 2 \leq i \leq s+1, j=i-1 \\ 1-\mathrm{e}^{-\mu T} & i=0, j=Q ; 2 \leq i \leq s+1, j=i+Q-1 \\ (1-p) \mathrm{e}^{-\mu T} & i=1, j=0 \\ (1-p)\left(1-\mathrm{e}^{-\mu T}\right) & i=1, j=Q \\ p \mathrm{e}^{-\mu T} & i=1, j=s \\ p\left(1-\mathrm{e}^{-\mu T}\right) & i=1, j=Q+s \\ 1 & s+2 \leq i \leq Q+s, j=i-1 \\ 0 & \text { otherwise }\end{cases}
$$

The stationary distribution of the embedded Markov chain is obtained by solving the equations $\pi(j)=\sum_{i} \pi(i) p(i, j)$ and the normalizing condition $\sum_{j} \pi(j)=1$. Using the function $p(i, j)$ given above we obtain, on solving $\pi(j)=\sum_{i} \pi(i) p(i, j)$,

$$
\begin{gathered}
\pi(j)=\frac{\left(\mathrm{e}^{\mu T}-1\right)}{(1-p)} \mathrm{e}^{(j-1) \mu T} \pi(0) ; \quad 1 \leq j \leq s \\
\pi(j)=\frac{\left(\mathrm{e}^{\mu T}-1\right)}{(1-p)} \mathrm{e}^{s \mu T} \pi(0)-\frac{p\left(\mathrm{e}^{\mu T}-1\right)}{(1-p)} \pi(0) ; \quad s+1 \leq j \leq Q \\
\pi(j)=\frac{\left(\mathrm{e}^{\mu T}-1\right)}{(1-p)} \mathrm{e}^{s \mu T} \pi(0)-\frac{p\left(\mathrm{e}^{\mu T}-1\right)}{(1-p)} \mathrm{e}^{(j-Q-1) \mu T} \pi(0) ; Q+1 \leq j \leq Q+s
\end{gathered}
$$

Using Equations (3.13) to (3.15) in $\sum_{j} \pi(j)=1$, we obtain

$$
\pi(0)=\frac{1}{1+\frac{Q\left(\mathrm{e}^{\mu T}-1\right)}{1-p} \mathrm{e}^{s \mu T}+\frac{(Q-s) p\left(\mathrm{e}^{\mu T}-1\right)}{1-p}}
$$

In order to determine the steady state inventory level distribution, we next determine the function $K(i, j, t)$ given by $K(i, j, t)=P\left[I(t)=j, \tau_{1}>t \mid I_{0}=i\right]$. Hence for $T>t$, we have

$$
K(i, j, t)= \begin{cases}\mathrm{e}^{-\mu t} & i=j=0 ; 2 \leq i \leq s+1, j=i-1 \\ 1-\mathrm{e}^{-\mu t} & i=0, j=Q ; 2 \leq i \leq s+1, j=i+Q-1 \\ (1-p) \mathrm{e}^{-\mu t} & i=1, j=0 \\ (1-p)\left(1-\mathrm{e}^{-\mu t}\right) & i=1, j=Q \\ p \mathrm{e}^{-\mu t} & i=1, j=s \\ p\left(1-\mathrm{e}^{-\mu t}\right) & i=1, j=Q+s \\ 1 & s+2 \leq i \leq Q+s, j=i-1 \\ 0 & \text { otherwise }\end{cases}
$$


and

$$
\begin{aligned}
& K^{*}(i, j, 0) \\
& = \begin{cases}\left(1-\mathrm{e}^{-\mu T}\right) / \mu & i=j=0 ; 2 \leq i \leq s+1, j=i-1 \\
T-\left(1-\mathrm{e}^{-\mu T}\right) / \mu & i=0, j=Q ; 2 \leq i \leq s+1, j=i+Q-1 \\
(1-p)\left(1-\mathrm{e}^{-\mu T}\right) / \mu & i=1, j=0 \\
(1-p) T-(1-p)\left(1-\mathrm{e}^{-\mu T}\right) / \mu & i=1, j=Q \\
p\left(1-\mathrm{e}^{-\mu T}\right) / \mu & i=1, j=s \\
p T-p\left(1-\mathrm{e}^{-\mu T}\right) / \mu & i=1, j=Q+s \\
T & s+2 \leq i \leq Q+s, j=i-1 \\
0 & \text { otherwise }\end{cases}
\end{aligned}
$$

Substituting for $K^{*}(i, j, 0)$ and $\pi(j)$ from (3.18) and (3.13) to (3.15) in (3.4) we obtain

$$
\begin{gathered}
P(0)=\frac{\left(\mathrm{e}^{\mu T}-1\right) \pi(0)}{\mu T} \\
P(j)=\frac{\left(\mathrm{e}^{\mu T}-1\right)^{2}}{\mu T(1-p)} \mathrm{e}^{(j-1) \mu T} \pi(0) ; 1 \leq j \leq s \\
P(j)=\frac{\left(\mathrm{e}^{\mu T}-1\right)}{(1-p)} \mathrm{e}^{s \mu T} \pi(0)-\frac{p\left(\mathrm{e}^{\mu T}-1\right)}{(1-p)} \pi(0) ; \quad s+1 \leq j \leq Q-1 \\
P(Q)=\frac{\left(\mathrm{e}^{\mu T}-1\right)}{(1-p)} \mathrm{e}^{s \mu T} \pi(0)-\frac{p\left(\mathrm{e}^{\mu T}-1\right)}{(1-p)} \pi(0)+\pi(0)-\frac{\left(\mathrm{e}^{\mu T}-1\right) \pi(0)}{\mu T} \\
P(j)=\frac{\left(\mathrm{e}^{\mu T}-1\right)}{(1-p)} \mathrm{e}^{s \mu T} \pi(0)-\frac{p\left(\mathrm{e}^{\mu T}-1\right)^{2}}{\mu T(1-p)} \mathrm{e}^{(j-Q-1) \mu T} \pi(0) ; Q+1 \leq j \leq Q+s
\end{gathered}
$$

Now that we have obtained the steady state inventory level distribution and the stationary probabilities of the embedded Markov chain, we can determine the various operating characteristics required to derive the cost function.

\section{Cost Function Derivation}

In this section we will deal with the problem of minimizing the total expected cost rate. We will also determine conditions under which it is cost optimal to place an emergency order with the local supplier rather than just wait for the order from your regular supplier. We use the following cost components

$K_{1}$ : the set-up cost per order for the regular order.

$K_{2}$ : the set-up cost per order for the emergency order. This cost is incurred whether or not the order is filled.

$c_{1}$ : the cost per item for the regular order.

$c_{2}$ : the cost per item for the emergency order. This cost is only incurred if the order is filled. 
$g$ : the shortage cost/unit short.

$h$ : the inventory carrying cost/unit/unit time.

Then the total expected cost rate is given by

$$
C(s, Q)=\left(K_{1}+c_{1} Q\right) \Gamma_{1}+\left(K_{2}+c_{2} s p\right) \Gamma_{2}+h \Gamma_{3}+g \Gamma_{4}
$$

where $\Gamma_{1}$ is the reorder rate for the regular order, $\Gamma_{2}$ is the reorder rate for the emergency order $\Gamma_{3}$ is the average inventory level and $\Gamma_{4}$ is the shortage rate.

A regular order is placed when the inventory level is $s+1$ and a demand occurs which brings the level down to the reorder point $s$. This order has a lead time which is exponentially distributed with a mean of $1 / \mu$. Hence

$$
\Gamma_{1}=\pi(s+1) / T
$$

An emergency order is placed when the inventory level is $n+1$ and a demand occurs or in the equivalent (instantaneous replenishment) system when the inventory level is one and a demand occurs. Hence

$$
\Gamma_{2}=\pi(1) / T
$$

The average inventory level

$$
\Gamma_{3}=\sum_{j} P(j)
$$

A shortage occurs when inventory level is zero and a demand occurs. Hence

$$
\Gamma_{4}=\pi(0) / T
$$

Substituting for $\Gamma_{1}, \Gamma_{2}, \Gamma_{3}$ and $\Gamma_{4}$ from (4.2) to (4.5) in (4.1) we obtain

$$
\begin{aligned}
C(s, Q)= & \left(K_{1}+c_{1} Q\right) \pi(s+1) / T+\left(K_{2}+c_{2} s[1-P(0)]\right) \pi(1) / T \\
& +h \sum_{j} P(j)+g \pi(0) / T
\end{aligned}
$$

Note that the fixed cost of placing an emergency order is incurred whether or not the order is satisfied while the variable cost is incurred only if the order is met. If the emergency order materializes, the inventory level does not touch zero. Hence probability that the variable cost of the emergency order is incurred is the probability the inventory level does not reach zero.

$C(s, Q)$ can be obtained explicitly by substituting for $\pi(j)$ s from Equations (3.13) to (3.15) and for $P(j)$ s from (3.1) to (3.5).

For a fixed $s$, the cost function is convex in $Q$ and for a fixed $Q$, it is convex in $s$. Although we were unable to prove the convexity of the cost function in two variables, our experience with various numerical examples indicates that the cost function is convex.

The long-run expected cost rate for the $(s, Q)$ that system with no emergency orders is given by

$$
C(s, Q)=\left(K_{1}+c_{1} Q\right) \Gamma_{5}+h \Gamma_{6}+g \Gamma_{7}
$$

Note that the only difference between Equation (4.7) and Equation (4.1) is the absence of the cost term for the emergency orders. The reorder rate, $\Gamma_{5}$, can be obtained by substituting $p=0$ in $\Gamma_{1}$. The average inventory level, $\Gamma_{6}$, can be obtained by substituting $p=0$ in $\Gamma_{3}$. The shortage rate, $\Gamma_{7}$, can be obtained by subs- 
tituting $p=0$ in $\Gamma_{4}$.

\section{Numerical Illustration}

In this section we compare the long-run expected cost rate of the system using emergency orders with that of the with standard lost sales $(s, Q)$ policy and establish cases where the system with emergency orders yields lower total costs or lower average inventory levels or both lower total costs and lower average inventory levels. For purposes of numerical illustration we assumed that the order size for the regular order $Q$ is bounded above by the size of the container. We used 200 as the container size. In order to determine the optimal parameters $s$ and $Q$, we do a complete enumeration and determine the pair $(s, Q)$ that gives us the minimum cost. The values held fixed in our numerical analysis were $K_{1}=$ 140, $K_{2}=70, c_{1}=20, T=0.02, \mu=2$. We then considered six values of $c_{2}$, ten values of $g$, two values of $h$, and 13 values of $p$. Our numerical analysis covered all possible combination of those values; i.e., 1560 combinations. Tables 1-6 tabulate the key results.

Table 1 and Table 2 address the question of how the inventory policy parameters $(s, Q)$ and average inventory are affected by using the emergency ordering system at the extreme value combinations of $c_{2}, g$ and $h$ for the various values of $p$. The tables show the results only for factor combinations where the long-run expected cost rate is lower for the emergency ordering than for the

Table 1. Illustrative impacts of emergency order system on policy parameters if holding cost $=1$.

\begin{tabular}{ccccccc}
\hline & \multicolumn{2}{c}{$g=40$} & \multicolumn{2}{c}{$g=180$} & \multicolumn{2}{c}{$g=50$} \\
\cline { 2 - 7 } $\boldsymbol{P}$ & \multicolumn{3}{c}{$c_{2}=22$} & \multicolumn{2}{c}{$c_{2}=180$} \\
\cline { 2 - 7 } & $(Q, s)$ & $\begin{array}{c}\text { Average } \\
\text { Inventory }\end{array}$ & $(Q, s)$ & $\begin{array}{c}\text { Average } \\
\text { Inventory }\end{array}$ & $(Q, s)$ & $\begin{array}{c}\text { Average } \\
\text { Inventory }\end{array}$ \\
\hline 0.00 & $(141,48)$ & 96 & $(146,99)$ & 148 & $(146,99)$ & 148 \\
0.10 & 75 & 152 & 106 & 148 & 117 & 149 \\
0.20 & 73 & 149 & 104 & 147 & 115 & 149 \\
0.40 & 70 & 142 & $(144,98)$ & 147 & 110 & 148 \\
0.50 & 68 & 138 & $(142,94)$ & 143 & 108 & 147 \\
0.60 & 66 & 133 & $(140,89)$ & 137 & 105 & 147 \\
0.70 & 63 & 127 & $(137,84)$ & 132 & 102 & 145 \\
0.80 & 60 & 121 & $(132,78)$ & 125 & 98 & 144 \\
0.90 & 56 & 113 & $(123,69)$ & 114 & 94 & 142 \\
0.95 & 54 & 108 & $(116,62)$ & 106 & 91 & 142 \\
0.98 & 52 & 105 & $(109,56)$ & 99 & 89 & 141 \\
0.99 & $(104,52)$ & 94 & $(106,54)$ & 96 & $(140,89)$ & 139 \\
1.00 & $(102,51)$ & 93 & $(102,51)$ & 93 & $(140,88)$ & 138 \\
\hline
\end{tabular}

Note: The shaded cells correspond to scenarios where emergency sourcing is inferior to single sourcing. 
Table 2. Illustrative impacts of emergency order system on policy parameters if holding cost $=10$.

\begin{tabular}{|c|c|c|c|c|c|c|}
\hline \multirow{3}{*}{$P$} & \multicolumn{2}{|c|}{$\boldsymbol{g}=40$} & \multicolumn{2}{|c|}{$g=180$} & \multirow{2}{*}{\multicolumn{2}{|c|}{$\begin{array}{c}g=50 \\
\boldsymbol{c}_{2}=180\end{array}$}} \\
\hline & \multicolumn{4}{|c|}{$c_{2}=22$} & & \\
\hline & $(Q, s)$ & $\begin{array}{l}\text { Average } \\
\text { Inventory }\end{array}$ & $(Q, s)$ & $\begin{array}{l}\text { Average } \\
\text { Inventory }\end{array}$ & $(Q, s)$ & $\begin{array}{l}\text { Average } \\
\text { Inventory }\end{array}$ \\
\hline 0.00 & $(51,15)$ & 27 & $(67,60)$ & 70 & $(67,60)$ & 70 \\
\hline 0.10 & 1 & 55 & 63 & 68 & 68 & 73 \\
\hline 0.20 & 13 & 50 & 62 & 65 & 67 & 70 \\
\hline 0.40 & 25 & 42 & $(59,58)$ & 65 & 64 & 65 \\
\hline 0.50 & 28 & 37 & $(57,56)$ & 62 & 62 & 63 \\
\hline 0.60 & 29 & 33 & $(54,53)$ & 58 & 60 & 61 \\
\hline 0.70 & 28 & 29 & $(50,49)$ & 53 & $(58,57)$ & 64 \\
\hline 0.80 & 26 & 27 & $(45,44)$ & 47 & $(55,54)$ & 60 \\
\hline 0.90 & $(25,24)$ & 22 & $(38,37)$ & 38 & $(51,50)$ & 55 \\
\hline 0.95 & $(24,23)$ & 22 & $(32,31)$ & 31 & $(48,47)$ & 51 \\
\hline 0.98 & $(23,22)$ & 21 & $(27,26)$ & 25 & $(46,45)$ & 49 \\
\hline 0.99 & $(23,22)$ & 21 & $(25,24)$ & 23 & $(45,44)$ & 48 \\
\hline 1.00 & $(22,21)$ & 20 & $(22,21)$ & 20 & $(44,43)$ & 46 \\
\hline
\end{tabular}

Note: The shaded cells correspond to scenarios where emergency sourcing is inferior to single sourcing.

Table 3. Minimum emergency order receipt probabilities for dual sourcing to be beneficial if holding cost $=1$.

\begin{tabular}{|c|c|c|c|c|c|c|}
\hline \multirow{2}{*}{$\boldsymbol{g}$ (shortage cost) } & \multicolumn{6}{|c|}{$c_{2}($ cost per unit for emergency sourcing) } \\
\hline & 22 & 25 & 27 & 30 & 40 & 50 \\
\hline 40 & 0.99 & $\mathrm{X}$ & $\mathrm{X}$ & $\mathrm{X}$ & $\mathrm{X}$ & $\mathrm{X}$ \\
\hline 50 & 0.90 & 1.00 & $\mathrm{X}$ & $\mathrm{X}$ & $\mathrm{X}$ & $\mathrm{X}$ \\
\hline 60 & 0.80 & 0.90 & 0.95 & $\mathrm{X}$ & $\mathrm{X}$ & $\mathrm{X}$ \\
\hline 70 & 0.80 & 0.80 & 0.90 & 0.95 & $\mathrm{X}$ & $\mathrm{X}$ \\
\hline 80 & 0.70 & 0.70 & 0.80 & 0.90 & $\mathrm{X}$ & $\mathrm{X}$ \\
\hline 90 & 0.70 & 0.70 & 0.80 & 0.90 & $\mathrm{X}$ & $\mathrm{X}$ \\
\hline 100 & 0.60 & 0.70 & 0.70 & 0.80 & $\mathrm{X}$ & $\mathrm{X}$ \\
\hline 120 & 0.60 & 0.60 & 0.70 & 0.70 & 0.95 & $\mathrm{X}$ \\
\hline 150 & 0.50 & 0.60 & 0.60 & 0.70 & 0.80 & 0.99 \\
\hline 180 & 0.40 & 0.50 & 0.50 & 0.60 & 0.80 & 0.90 \\
\hline
\end{tabular}

Note: $\mathrm{X}=$ single sourcing is always cheaper than emergency sourcing for that combination of $g$ and $c_{2}$. 
Table 4. Minimum emergency order receipt probabilities for dual sourcing to be beneficial if holding cost $=10$.

\begin{tabular}{|c|c|c|c|c|c|c|}
\hline \multirow{2}{*}{$\boldsymbol{g}$ (shortage cost) } & \multicolumn{6}{|c|}{$c_{2}$ (cost per unit for emergency sourcing) } \\
\hline & 22 & 25 & 27 & 30 & 40 & 50 \\
\hline 40 & 0.90 & 1.00 & $\mathrm{X}$ & $\mathrm{X}$ & $\mathrm{X}$ & $\mathrm{X}$ \\
\hline 50 & 0.80 & 0.90 & 0.90 & 0.99 & $\mathrm{X}$ & $X$ \\
\hline 60 & 0.70 & 0.80 & 0.80 & 0.90 & $\mathrm{X}$ & $\mathrm{X}$ \\
\hline 70 & 0.60 & 0.70 & 0.80 & 0.80 & $\mathrm{X}$ & $\mathrm{X}$ \\
\hline 80 & 0.60 & 0.60 & 0.70 & 0.80 & 0.95 & $\mathrm{X}$ \\
\hline 90 & 0.50 & 0.60 & 0.60 & 0.70 & 0.90 & $\mathrm{X}$ \\
\hline 100 & 0.50 & 0.60 & 0.60 & 0.70 & 0.80 & 0.98 \\
\hline 120 & 0.40 & 0.50 & 0.50 & 0.60 & 0.70 & 0.90 \\
\hline 150 & 0.40 & 0.40 & 0.50 & 0.50 & 0.60 & 0.80 \\
\hline 180 & 0.40 & 0.40 & 0.40 & 0.40 & 0.60 & 0.70 \\
\hline
\end{tabular}

Note: $\mathrm{X}=$ single sourcing is always cheaper than emergency sourcing for that combination of $g$ and $c_{2}$.

Table 5. Percentage cost reduction achieved with dual sourcing for guaranteed emergency order receipt if holding cost $=1$.

\begin{tabular}{|c|c|c|c|c|c|c|}
\hline \multirow{2}{*}{$\boldsymbol{g}$ (shortage cost) } & \multicolumn{6}{|c|}{$\boldsymbol{c}_{2}$ (cost per unit for emergency sourcing) } \\
\hline & 22 & 25 & 27 & 30 & 40 & 50 \\
\hline 40 & $0.09 \%$ & $\mathrm{X}$ & $\mathrm{X}$ & $\mathrm{X}$ & $\mathrm{X}$ & $\mathrm{X}$ \\
\hline 50 & $0.95 \%$ & $0.17 \%$ & $\mathrm{X}$ & $\mathrm{X}$ & $\mathrm{X}$ & $\mathrm{X}$ \\
\hline 60 & $1.56 \%$ & $0.78 \%$ & $0.35 \%$ & $\mathrm{X}$ & $\mathrm{X}$ & $\mathrm{X}$ \\
\hline 70 & $2.02 \%$ & $1.25 \%$ & $0.82 \%$ & $0.27 \%$ & $\mathrm{X}$ & $\mathrm{X}$ \\
\hline 80 & $2.40 \%$ & $1.63 \%$ & $1.20 \%$ & $0.66 \%$ & $\mathrm{X}$ & $\mathrm{X}$ \\
\hline 90 & $2.71 \%$ & $1.95 \%$ & $1.52 \%$ & $0.98 \%$ & $\mathrm{X}$ & $\mathrm{X}$ \\
\hline 100 & $2.99 \%$ & $2.22 \%$ & $1.80 \%$ & $1.26 \%$ & $\mathrm{X}$ & $\mathrm{X}$ \\
\hline 120 & $3.44 \%$ & $2.68 \%$ & $2.26 \%$ & $1.72 \%$ & $0.40 \%$ & $\mathrm{X}$ \\
\hline 150 & $3.96 \%$ & $3.21 \%$ & $2.79 \%$ & $2.25 \%$ & $0.94 \%$ & $0.04 \%$ \\
\hline 180 & $4.37 \%$ & $3.62 \%$ & $3.20 \%$ & $2.67 \%$ & $1.36 \%$ & $0.47 \%$ \\
\hline
\end{tabular}

Note: $\mathrm{X}=$ single sourcing is always cheaper than emergency sourcing for that combination of $g$ and $c_{2}$.

Table 6. Percentage cost reduction achieved with dual sourcing for guaranteed emergency order receipt if holding cost $=10$.

\begin{tabular}{|c|c|c|c|c|c|c|}
\hline \multirow{2}{*}{$\boldsymbol{g}$ (shortage cost) } & \multicolumn{6}{|c|}{$c_{2}$ (cost per unit for emergency sourcing) } \\
\hline & 22 & 25 & 27 & 30 & 40 & 50 \\
\hline 40 & $3.89 \%$ & $0.12 \%$ & $\mathrm{X}$ & $\mathrm{X}$ & $\mathrm{X}$ & $\mathrm{X}$ \\
\hline 50 & $9.04 \%$ & $5.48 \%$ & $3.30 \%$ & $0.30 \%$ & $\mathrm{X}$ & $\mathrm{X}$ \\
\hline 60 & $12.57 \%$ & $9.14 \%$ & $7.05 \%$ & $6.66 \%$ & $\mathrm{X}$ & $\mathrm{X}$ \\
\hline 70 & $15.21 \%$ & $11.89 \%$ & $9.86 \%$ & $7.07 \%$ & $\mathrm{X}$ & $\mathrm{X}$ \\
\hline 80 & $17.29 \%$ & $14.05 \%$ & $12.07 \%$ & $9.34 \%$ & $2.04 \%$ & $\mathrm{X}$ \\
\hline 90 & $18.99 \%$ & $15.81 \%$ & $13.87 \%$ & $11.21 \%$ & $4.06 \%$ & $\mathrm{X}$ \\
\hline 100 & $20.42 \%$ & $17.30 \%$ & $15.40 \%$ & $12.78 \%$ & $5.75 \%$ & $0.50 \%$ \\
\hline 120 & $22.72 \%$ & $19.69 \%$ & $17.84 \%$ & $15.30 \%$ & $8.47 \%$ & $3.38 \%$ \\
\hline 150 & $25.28 \%$ & $22.35 \%$ & $20.56 \%$ & $18.10 \%$ & $11.51 \%$ & $6.58 \%$ \\
\hline 180 & $27.19 \%$ & $24.34 \%$ & $22.60 \%$ & $20.20 \%$ & $13.78 \%$ & $8.98 \%$ \\
\hline
\end{tabular}

Note: $\mathrm{X}=$ single sourcing is always cheaper than emergency sourcing for that combination of $g$ and $c_{2}$. 
standard lost sales $(s, Q)$ policy. Thus, the combination of $\left(c_{2}, g\right)=(50,40)$ is excluded because the standard lost sales $(s, Q)$ policy for that combination was superior to the emergency order policy at every value of $p$. The tables confirm the expected outcome that the emergency order policy can operate optimally with smaller inventories than the standard lost sales $(s, Q)$ policy. For example, at $\left(c_{2}\right.$, $g, h)=(22,180,10)$, the buyer's average inventory level when the option of emergency ordering is unavailable is 70 units but drops to less than one-third of that amount (20 units) when emergency order fulfillment (receipt) is guaranteed; i.e. if $p=1$.

Table 3 and Table 4 focus on determining the minimum emergency order receipt probability $(p)$ that must be reached in order for dual sourcing to yield a lower long-run expected cost rate than the standard lost sales $(s, Q)$ policy. Consider, as illustration, the column for $c_{2}=50$ in Table 1. This shows that unless the shortage cost exceeds a value somewhere in the range $120 \leq g<150$, dual sourcing does not make sense. Further, even if that condition is satisfied, the dual sourcing is advantageous only if the probability of order fulfilment by the emergency source is high. Case in point is that at $g=150$, the probability must reach 0.99 . At the higher value of $g=180$, the required probability threshold is less (0.90). The threshold order fulfilment probability requirements are lower (or at least, non-increasing) for lower unit prices of emergency ordered items $\left(c_{2}\right)$ and for higher unit costs of holding inventory $(h)$.

These observations have important sourcing policy implications. In particular, as noted earlier, emergency order fulfilment by infrequently used domestic suppliers is unlikely to have a high probability, much less be guaranteed. As such, an emergency order policy might have to be complemented by a policy of ordering more frequently from the domestic supplier; i.e., order even in the absence of an emergency requirement. That way, the domestic supplier may accord the buyer's order the high priority given to orders from its regular customers and thus be more inclined to fill the emergency order. Clearly, this means higher long-run inventory cost rate and requires the buyer to determine the revised order frequency from a domestic source would assure a sufficiently high order fulfilment probability. Analysis of this emergency ordering tactic is beyond the scope of this paper and is considered as a matter for possible future research.

Table 5 or Table 6 show the percentage cost reductions of using the emergency ordering policy instead of the standard lost sales $(s, Q)$ policy, under the assumption of guaranteed order fulfillment (receipt). The reported percentages follow the expected directions: they are higher for 1 ) lower values of $c_{2}$ (lower item cost penalty of using the emergency source), 2) higher values of $g$ (avoidance of larger stockout penalties by using the emergency source), and 3) higher values of $h$ (higher savings from the reduced inventory levels when the emergency source is used). For low holding cost ( $h=1 \%$ or $5 \%$ of item price from regular supplier), the best improvement observed was a very moderate $4.37 \%$. The increase of $h$ to 10 (50\% of the item price from regular supplier) yields more striking improvements. These improvements can be viewed as the premium a 
company would be willing to pay to have guaranteed insurance against stockouts.

These observations may offer some insights into the strategies used by some companies to handle their US-Canada trans-border supply chain operations. To explain these insights, we first note that firms such as automobile manufactures might face scenarios closer to the lower rows of Table 6 than to any of the other scenarios in either Table 5 or Table 6 . Specifically, because these firms use just-in-time delivery, failure of a just-in-time order of parts to arrive as scheduled (a stockout) can cause costly shut down of a day's scheduled production. The fact that they use just-in-time as an inventory reduction strategy leads one to deduce that their inventory carrying costs are high enough to be warrant such a strategy. Indeed, holding cost data used for the models in Nozick and Turnquist (2001) support that deduction. These points make it understandable that these firms have been willing to pay what is regarded as a high premium for insurance against stockouts. However, the insurance that these firms have procured is more preventive rather than remedial (as is emergency sourcing). That insurance involves investing in supply chain security initiatives that are necessary for firms to receive expedited border checkpoint processing of their trans-border shipments.

The derivable insights from these results apply beyond the automobile manufacturing sector. Since, as we have noted, the values in Table 5 and Table 6 provide a measure of what can be gained from having guaranteed inventory availability, they suggest what may be defensible levels of investment to have that guarantee. Thus, for a buyer facing the scenario in the last row of Table 5 under the column for $c_{2}=22$ an investment limit of $4.37 \%$ of the expected cost rate for regular orders might be appropriate since the total cost is lowered by $4.37 \%$ when emergency ordering is used (see Table 5). That sort of guideline might be useful for practical decisions of how much to invest in supply chain security initiatives that promise such guarantee; e.g., initiatives such as the Customs-Trade Partnership Against Terrorism (C-TPAT).

\section{Conclusions}

Using a lost sales context, we study a dual sourcing policy that uses emergency ordering when a delay in the order from the regular source will lead to an imminent stockout. We model the policy as a lost sales $(s, Q)$ system. By demonstrating its equivalence to systems with instantaneous emergency order delivery (with a known delivery probability) we overcome some mathematical complexities of modeling continuous review systems. Through an extensive set of numerical examples, we find that complementing regular orders with emergency orders does not always reduce total costs (comprising ordering cost, inventory carrying cost, shortage cost, and item cost). We identify conditions under which this dual sourcing policy yields lower total costs than the standard lost sales $(s$, $Q)$ policy without emergency. Further, we quantify the magnitude of the cost 
reductions if delivery of the emergency order is guaranteed; i.e., guaranteed elimination of shortages.

A key conclusion from our work is that the dual sourcing policy might have to be supported by companion strategies to provide that guarantee. The results provided some insights into known company behaviour in the empirical context that motivated the study: US-Canada trans-border supply chains. That is, we surmise that the reasons firms such as automobile manufacturers pursue strategies aimed at guaranteed elimination of shortages might be the resulting large total cost reductions. Two items we see as potential future research goals are 1) developing closed-form solutions to readily produce the paper's results and insights without reliance on extensive numerical examples and 2) studying policies that tolerate some increase in inventory holding costs in order to have guarantees that the emergency source will deliver the emergency order.

\section{Acknowledgements}

This work was funded by the SSHRC grant of the authors. The authors would like to thank the referees for their feedback which significantly improved the presentation of the paper.

\section{Conflicts of Interest}

The authors declare no conflicts of interest regarding the publication of this paper.

\section{References}

[1] Thomas, D.J. and Tyworth, J.E. (2007) Is Pooling Lead-Time Risk by Splitting Orders Simultaneously Worthwhile? Journal of Business Logistics, 28, 169-193. https://doi.org/10.1002/j.2158-1592.2007.tb00236.x

[2] Minner, S. (2003) Multiple Supplier Inventory Models in Supply Chain Management: A Review. International Journal of Production Economics, 81-82, 265-279. https://doi.org/10.1016/S0925-5273(02)00288-8

[3] Barankin, E.W. (1961) A Delivery-Lag Inventory Model with Emergency Provision. Naval Research Logistics, 8, 285-311. https://doi.org/10.1002/nav.3800080310

[4] Neuts, M.F. (1964) An Inventory Model with an Optimal Time Lag. Journal of the Society for Industrial and Applied Mathematics, 12, 179-185. https://doi.org/10.1137/0112016

[5] Johansen, S.G. (2018) Emergency Orders in the Periodic-Review Inventory System with Fixed Ordering Costs and Stochastic Lead Times for Normal Orders. International Journal of Production Economics, in Press. https://doi.org/10.1016/j.ijpe.2018.01.017

[6] Dohi, T., Kaio, N. and Osaki, S. (1995) Continuous-Review Cyclic Inventory Models with Emergency Orders. Journal of the Operations Research Society of Japan, $\mathbf{3 8}$, 212-229. https://doi.org/10.15807/jorsj.38.212

[7] Giri, B.C. and Dohi, T. (2009) Cost-Effective Ordering Policies for Inventory Systems with Emergency Order. Computers \& Industrial Engineering, 57, 1336-1341. https://doi.org/10.1016/j.cie.2009.07.001 
[8] Bradley, J.R. (2004) A Brownian Approximation of a Production-Inventory System with a Manufacturer That Subcontracts. Operations Research, 52, 765-784. https://doi.org/10.1287/opre.1040.0135

[9] Allon, G. and Van Mieghem, J.A. (2010) Global Dual Sourcing: Tailored Base-Surge Allocation to Near- and Offshore Production. Management Science, 56, 110-124. https://doi.org/10.1287/mnsc.1090.1099

[10] Allen, S.G. and d'Esppo, D.A. (1968) An Ordering Policy for Stock Items When Delivery Can Be Expedited. Operations Research, 16, 880-883. https://doi.org/10.1287/opre.16.4.880

[11] Moinzadeh, K. and Schmidt, C.P. (1991) An (S - 1, S) Inventory System with Emergency Orders. Operations Research, 39, 308-321. https://doi.org/10.1287/opre.39.2.308

[12] Song, J.S. and Zipkin, P. (2009) Inventories with Multiple Supply Sources and Networks of Queues with Overflow Bypasses. Management Science, 55, 362-372. https://doi.org/10.1287/mnsc.1080.0941

[13] Johansen, S.G. and Thorstenson, A. (1998) An Inventory Model with Poisson Demands and Emergency Orders. International Journal of Production Economics, 56, 275-289. https://doi.org/10.1016/S0925-5273(97)00126-6

[14] Moinzadeh, K. and Nahmias, S. (1988) A Continuous Review Model for an Inventory System with Two Supply Modes. Management Science, 34, 761-773. https://doi.org/10.1287/mnsc.34.6.761

[15] Mohebbi, E. and Posner, M.J.M. (1999) A Lost Sales Continuous Review Inventory System with Emergency Ordering. International Journal of Production Economics, 58, 93-112. https://doi.org/10.1016/S0925-5273(98)00208-4

[16] Duran, A., Gutierrez, G. and Zequeira, R.I. (2004) A Continuous Review Inventory Model with Order Expediting. International Journal of Production Economics, 87, 157-169. https://doi.org/10.1016/S0925-5273(03)00100-2

[17] Axsäter, S. (2007) A Heuristic for Triggering Emergency Orders in an Inventory System. European Journal of Operational Research, 176, 880-891. https://doi.org/10.1016/j.ejor.2005.09.002

[18] Cinlar, E. (1975) Introduction to Stochastic Processes. Prentice Hall, Englewood Cliffs, NJ. 